\title{
Cardiac Monitoring to aid in Diagnosis
}

\author{
Manu Mitra \\ Department of Electrical Engineering, Alumnus of University of Bridgeport, Bridgeport, United States
}

\begin{abstract}
Cardiac Monitoring refers monitoring heart activity to determine patient's condition relative to their cardiac rhythm. The reason of having cardiac monitoring is to determine any unusual activity of heart; so that it can diagnosed before the severity condition of patient increases. There are many ways to determine condition of health of heart. There are many others, however, in this paper flexible and comfortable ways are discussed without inconvenience for the patient. For example - flexible device on the finger, flexible skin like monitor small enough size of a bandage and self-powered cardiac pacemaker using nanogenerator.
\end{abstract}

(C) 2020 Author(s). All rights reserved.

Keywords: Cardiac Monitoring, Diagnosis, Heart, Cardiac Telemetry, Flexible Cardiac Monitoring.

\section{Introduction}

Manuscripts should be written in English. Invited contributions may exceptionally be in Portuguese. This template for preparation of manuscripts for Cienc. Tecnol. Mater. should be followed.

Cardiac monitoring is used to determine heart rhythm disorder and to identify the right course of treatment. Various symptoms related to heart conditions that includes atrial fibrillation, unexplained stroke, heart palpitations, unexplained fainting. Advanced technologies makes feasible for doctors to get information about heart without interrupting life, it is useful in capturing irregular heartbeats and in some cases patient may not even feel any symptoms. Few of the monitoring systems include such as holter monitor, event recorder, Mobile Cardiac Telemetry (MCT), Insertable Cardiac Monitor (ICM) to monitor heart (Medtronic, 2018).
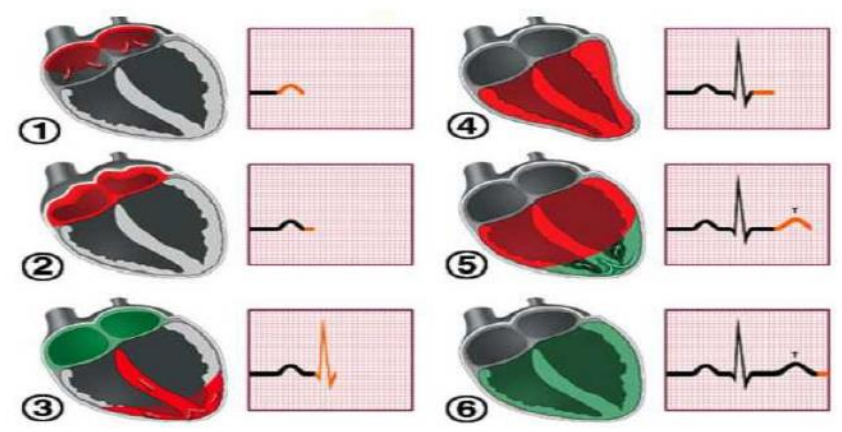

Fig. 1. Illustrates Heart behavior and part of the generated signal. Image Credit: Liena Elrayah Abdelkhair Khairelseed (Khairelseed, 2011)

\footnotetext{
* Corresponding author.

E-mail address: mmitra@my.bridgeport.edu (Manu Mitra)
} 


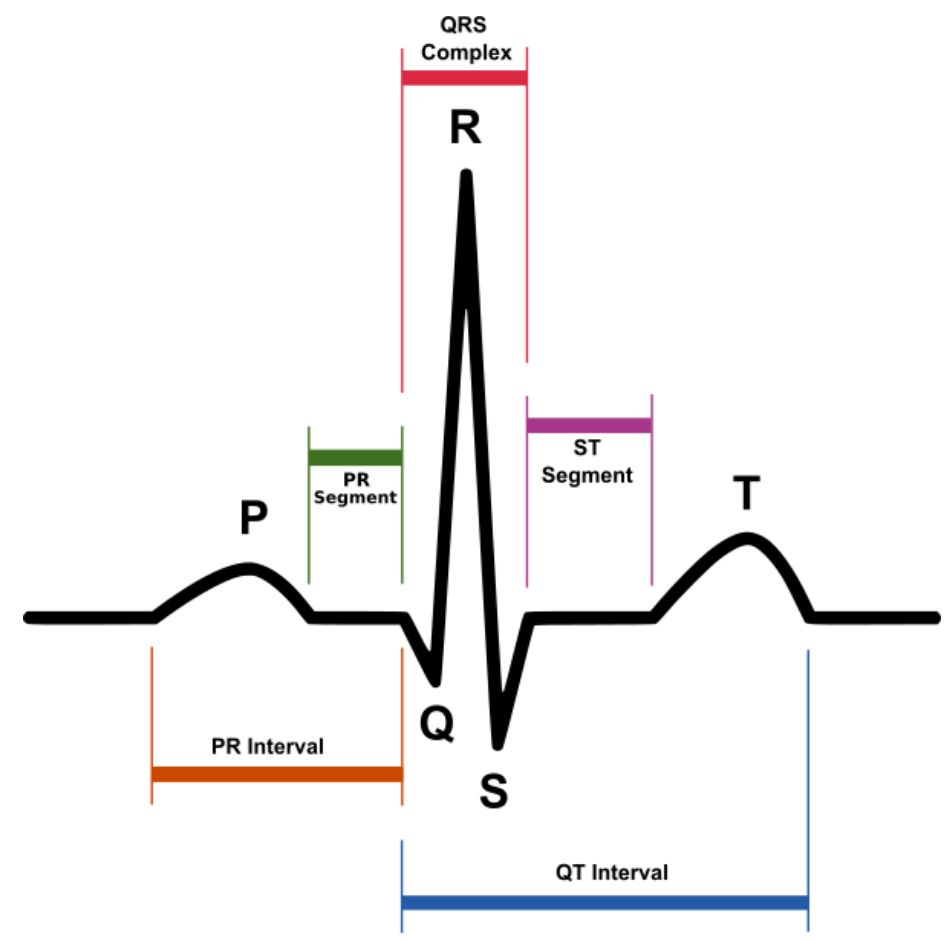

Fig. 2. Illustrates $\mathrm{ECG}$ of a heart in normal sinus rhythm where $\mathrm{O}$ is the origin or datum point preceding the cycle. $\mathrm{P}$ is the atrial systole contraction pulse. $\mathrm{Q}$ is a downward deflection immediately preceding the ventricular contraction. $\mathrm{R}$ is the peak of the ventricular contraction. $\mathrm{S}$ is the downward deflection immediately after the ventricular contraction. $\mathrm{T}$ is the recovery of the ventricles. $\mathrm{U}$ is the successor of the $\mathrm{T}$ wave but it is small and not always observed (Wikipedia, 2018).

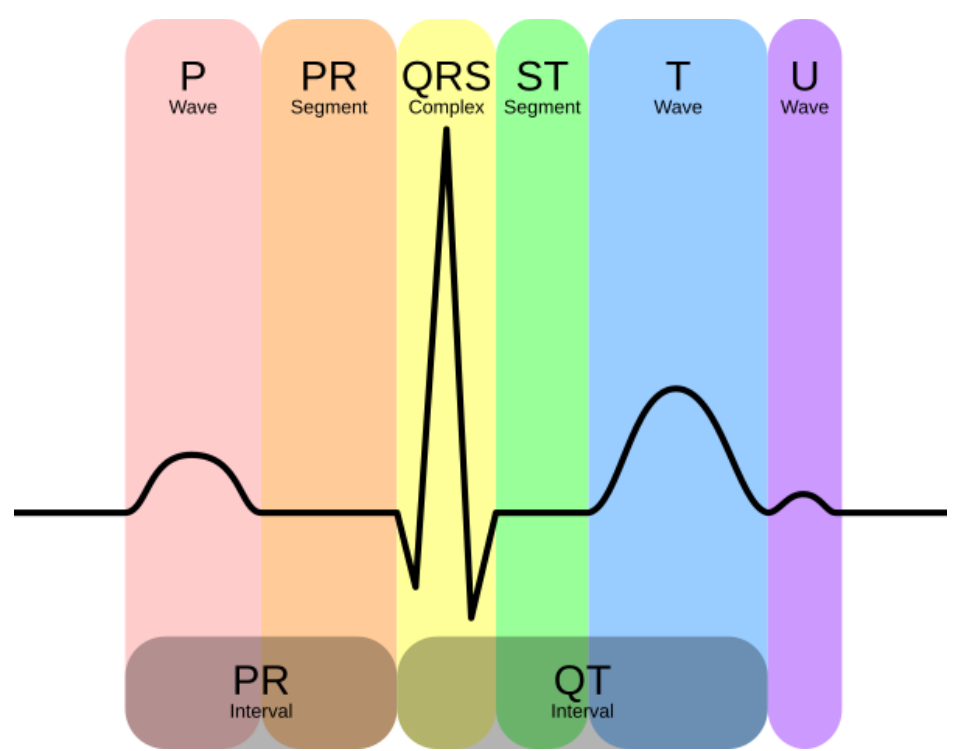

Fig. 3. Illustrates Schematic representation of a normal ECG. Normal rhythm produces four entities - a P wave, a QRS complex, a T wave, and a $\mathrm{U}$ wave - that each have a fairly unique pattern. The $\mathrm{P}$ wave represents atrial depolarization. The QRS complex represents ventricular depolarization. The $\mathrm{T}$ wave represents ventricular repolarization. The $\mathrm{U}$ wave represents papillary muscle repolarization (Wikipedia, 2018). 


\section{Monitoring Heart Through Self-powered Device}

Self-powered devices that is suitable directly on human skin or tissue would have great future for medical applications. On the other hand, practical understanding has been unworkable due to bulkiness of batteries, inadequate power supply, noise interference, impending conformability and long term operations. To evaluate a practical application, sensory devices called organic electrochemical transistors were incorporated with organic solar cells on an extremely thin substrate to allow self-powered detection of heartbeats to account electrocardiographic signals.

Scientist were able to design an advanced, user friendly, very flexible organic sensor powered by sunlight which operates as self-powered heart monitor. In this investigation results, they combined a sensory apparatus called an organic electrochemical transistor; it is a form of electronic device that can be used to measure variety of biological functions. Using this device scientists were able to measure the heart beats of humans and rats under bright light conditions.

They found that the device operated successfully at a lighting level of 10, 000 lux, which is equivalent to the light seen in the shade on a clear sunny day and experienced less noise than other devices connected to battery because of lack of electric wires

"This a step ahead in exploration to make self-powered medical monitoring devices that can be placed on human tissue" as per Kenhiro Fukuda of RIKEN Center for Emergent Matter Science (Park, 2018; RIKEN, 2008).

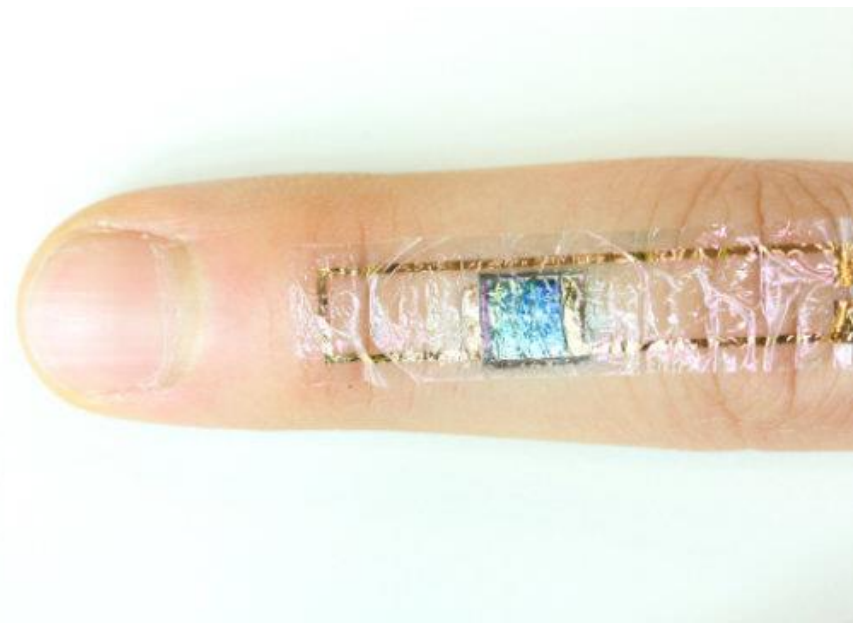

Fig. 4. Illustrates flexible device on a finger. Image Credit: Image courtesy of RIKEN (RIKEN, 2008)

\section{Heart Failure monitoring through a tiny apparatus}

Increasing in pressure with increase in weight and changes in blood pressure are symptoms of worsening of heart failure. It also allows patients to transmit daily readings from their home to their physician, for proactive management of their heart condition; an analysis of patients with Class III heart breakdown had admissions by 28 percent at 15 months. Class III heart failure is characterized by American Heart Association as patients with cardiac disease resulting in limiting of physical activity. They are happy when they are at rest. Less than ordinary activity may result in palpitation, fatigue, dyspnea or angina pain. Approximately 1.5 million Americans live with Class III heart breakdown. This device is the only heart failure monitoring approved by Food and Drug Administration (FDA). 
This new technology in implant gives patients the convenience to send daily updates about their condition of their heart to physicians. Apparatus is a size of a paper clip sensor that is implanted in the pulmonary artery to measure pulmonary artery pressure.

"This implant device was supposed to last for lifetime and the patient don't have to worry about changing batteries. It will help to give patients a better quality of life and significantly lessen on hospital admissions and re-admissions." was confirmed by Bhimiraj (Houston Methodist, 2014).

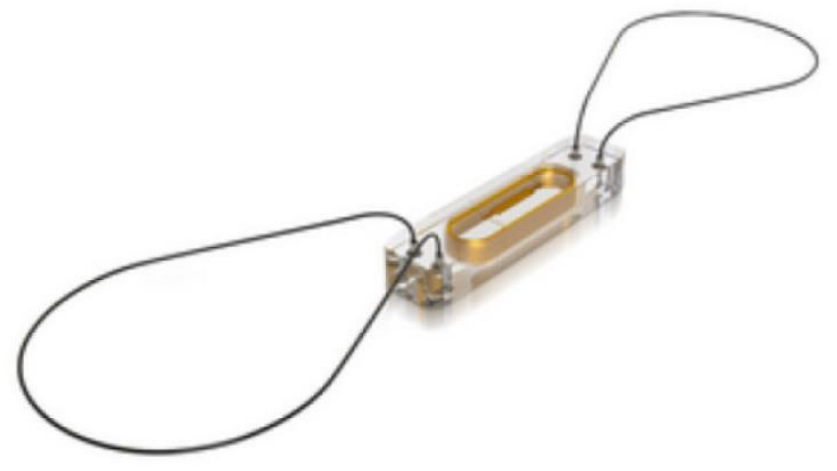

Fig. 5. Illustrates Cardio MEMES HF Systems Image Credit: Courtesy of St. Jude Medical Inc. (Houston Methodist, 2014)

\section{Heart Health Monitoring using thin Paper}

Scientists integrated various layers of flexible materials into pressure sensors to design a heart monitor which is thinner than a bandage. Prof. Zhenan Bao of chemical engineering at Stanford designed a heart monitor which is thinner than a dollar bill and less wide than a postage stamp. This flexible monitor device can be worn under adhesive bandage on the wrist and is perceptive enough to help doctors or physicians to identify stiff arteries and cardiovascular complications. These devices can be used to track continuous heart health and provide physicians a safer technique of measuring a very important sign for newborn and other high-risk surgery patients.

When pressure is applied on the apparatus, the pyramids deforms changing the size of the distance between the two halves of the apparatus. This alteration in separation causes a measurable change in the electromagnetic field and flow of current in the apparatus. When sensor is implanted one the wrist using an adhesive bandage, it can calculate that person's pulse wave as it reverberates through the body.

"The pulse is related to the condition of artery and state of the heart and the better the sensor the better physicians can get hold of complications before they develop" was said by Bao (Schwartz, et.al, 2013; Stanford University, 2013).

\section{Blood Pressure monitoring using wearable patch}

A new wearable ultrasound patch that is very flexible and monitors blood pressure in arteries that are deep beneath the skin that may help patients detect cardiovascular complications earlier and with better precision.

In experiments, the patch accomplished some clinical methods to calculate blood pressure. Functions of this device include real time and continuous intensive care of blood pressure changes in patients with heart or lung disease as well as for patients undergoing surgery. This patch uses ultrasound technique so that it could potentially track other vital signs and physiological signals from places which are deep inside the body. 
This device calculates central blood pressure which actually differs from the blood pressure taken from inflatable cuff strapped around the upper arm, which is known as peripheral blood pressure. Central blood pressure in the central blood vessels, which is send blood directly from the heart to other major organs throughout the body. Medical physicians consider central blood pressure more accurate than peripheral blood pressure and also more precise in predicting heart disease.

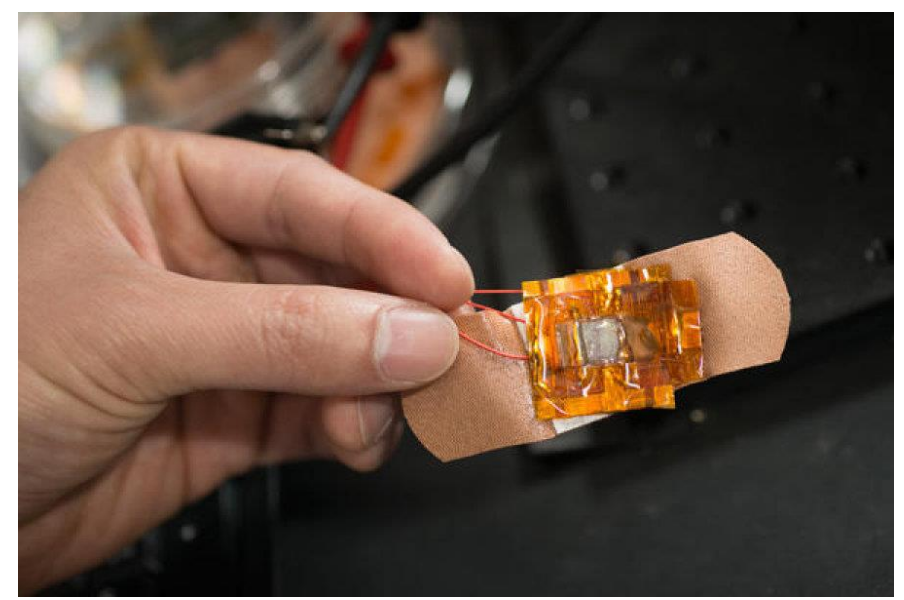

Fig. 5. Illustrates flexible skin-like heart monitor is small enough to wear under a bandage. Image Credit: L.A. Cicero (Stanford University, 2013)

A non-invasive technique does exists but it does not always produce accurate reading. It comprises holding a pen like probe called tonometer. To get good reading, the tonometer must be held steady at just right angle and right amount of pressure each time. But results vary between tests and different techniques. This research was led by University of California San Diego (Wang, et.al., 2018; University of California-San Diego, 2018).

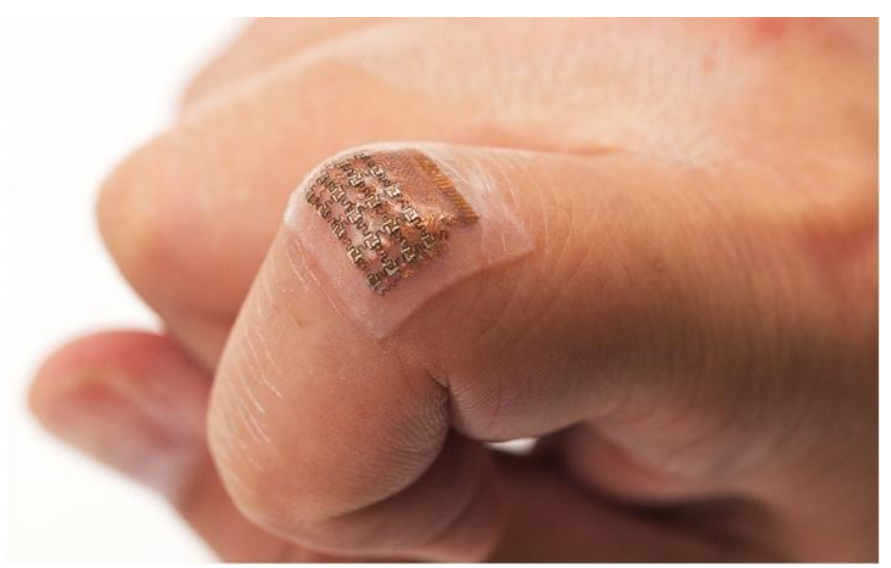

Fig. 6. Illustrates wearable ultrasound patch tracks blood pressure in a deep artery or vein. Image Credit: Chonghe Wang/Nature Biomedical Engineering (University of California-San Diego, 2018)

\section{Self-Powered Cardiac Pacemaker}

Researchers have developed a self-powered artificial cardiac pacemaker that can be conducted semi-permanently by a soft piezoelectric nanogenerator. 
This flexible piezoelectric nanogenerator directly stimulated a living rat's heart using electrical energy. This novel technique can aid the use of self-powered energy harvesters for not only lifetime of cardiac pacemakers but also in real time heart monitoring. This artificial cardiac pacemaker can be used as medical equipment that is combined into human body to control the heartbeats through electrical stimulation that contracts cardiac muscles who suffers from arrhythmia. On the other hand, repeated surgeries are to replace pacemaker. This high performance flexible nanogenerators make use of single crystal PMN-PT thin film (iBULe Photonics). The collected energy reached up to $8.2 \mathrm{~V}$ and $0.22 \mathrm{~mA}$ by bending and pushing motions which were high enough to directly stimulate the rat's heart.

"This flexible piezoelectric nanogenerator can be used as an electrical source for various implantable medical apparatus. This significant achievement will aid the development of self-powered cardiac pacemaker and may help to halt heart attacks via real-time diagnosis of heart arrhythmia" was said by Prof. Keon Jae Lee (Hwang, et.al., 2014; KAIST, 2014).

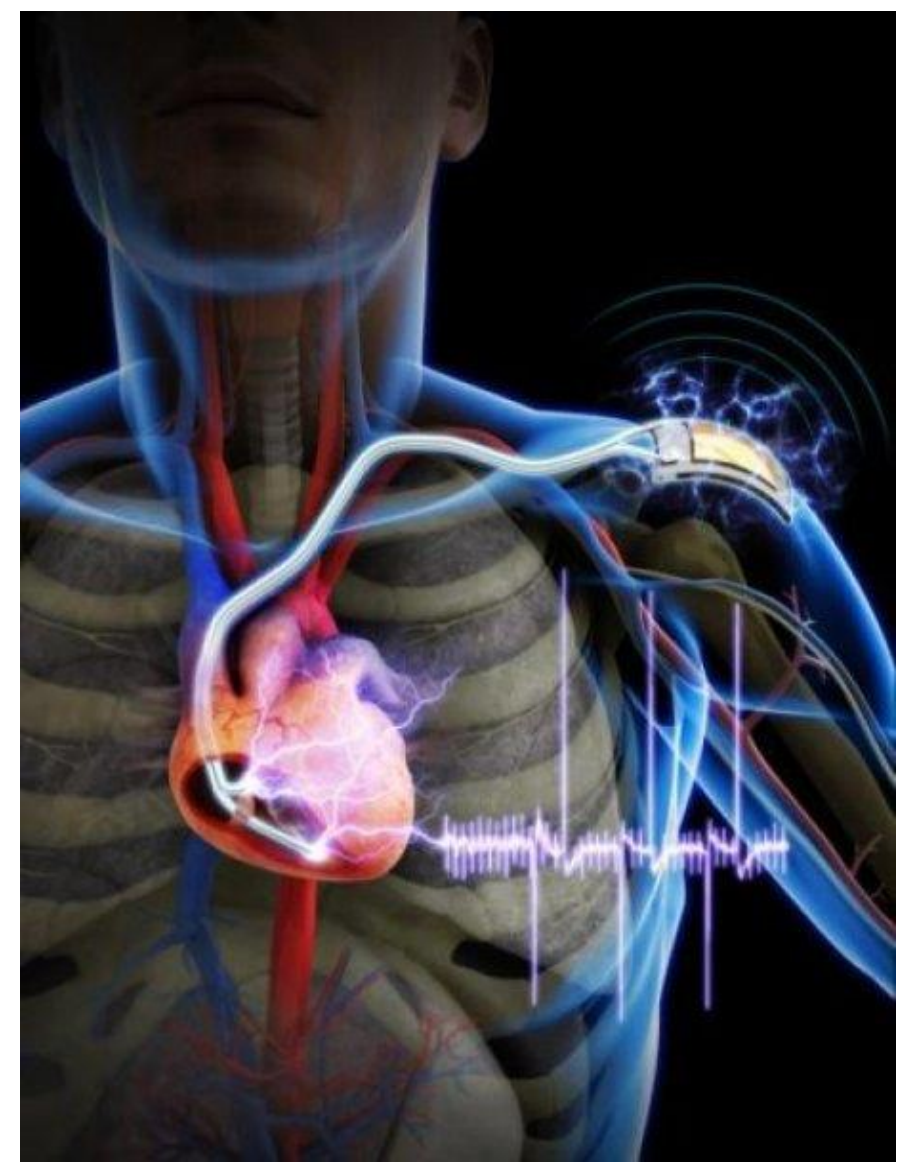

Fig. 7. Illustrates that a self-powered cardiac pacemaker is enabled by a flexible piezoelectric energy harvester. Image Credit: KAIST (KAIST, 2014)

\section{Acknowledgements}

Author would like to thank Prof. Navarun Gupta, Prof. Hassan Bajwa, Prof. Linfeng Zhang and Prof. Hmurcik for their academic support. Author also thanks anonymous reviewers for their comments. 


\section{Conflicts of Interest}

There are no conflict of interest as per Author's point of view.

\section{References}

Chonghe Wang, Xiaoshi Li, Hongjie Hu, Lin Zhang, Zhenlong Huang, Muyang Lin, Zhuorui Zhang, Zhenan Yin, Brady Huang, Hua Gong, Shubha Bhaskaran, Yue Gu, Mitsutoshi Makihata, Yuxuan Guo, Yusheng Lei, Yimu Chen, Chunfeng Wang, Yang Li, Tianjiao Zhang, Zeyu Chen, Albert P. Pisano, Liangfang Zhang, Qifa Zhou, Sheng Xu. (2018). Monitoring of the central blood pressure waveform via a conformal ultrasonic device. Nature Biomedical Engineering, 2 (9): 687 DOI: 10.1038/s41551-018-0287-x

Geon-Tae Hwang, Hyewon Park, Jeong-Ho Lee, SeKwon Oh, Kwi-Il Park, Myunghwan Byun, Hyelim Park, Gun Ahn, Chang Kyu Jeong, Kwangsoo No, HyukSang Kwon, Sang-Goo Lee, Boyoung Joung, Keon Jae Lee. (2014). Self-Powered Cardiac Pacemaker Enabled by Flexible Single Crystalline PMN-PT Piezoelectric Energy Harvester. Advanced Materials. DOI: 10.1002/adma.201400562

Gregor Schwartz, Benjamin C.-K. Tee, Jianguo Mei, Anthony L. Appleton, Do Hwan Kim, Huiliang Wang, Zhenan Bao. (2013). Flexible polymer transistors with high pressure sensitivity for application in electronic skin and health monitoring. Nature Communications. 4, 1859. DOI: 10.1038/ncomms2832

Houston Methodist. (2014, October 13). Monitoring heart failure with tiny implant. ScienceDaily. Retrieved December 11, 2018 from www.sciencedaily.com/releases/2014/10/141013130106.htm

Khairelseed, L. A. (2011). Micro controller based heart rate monitor using finger strip sensor (Master's thesis). Retrieved from http://khartoumspace.uofk.edu/bitstream/handle/123456789/18587/Microcontroller\%20Based\%20Heart\%20Rat e.pdf

Medtronic. (2018, July). Heart Monitoring. Retrieved from https://www.medtronic.com/us-en/patients/treatmentstherapies/heart-monitors/monitoring.html

RIKEN. (2018, September 26). A self-powered heart monitor taped to the skin. ScienceDaily. Retrieved December 11, 2018 from www.sciencedaily.com/releases/2018/09/180926140821.htm

Stanford University. (2013, May 16). Engineers monitor heart health using paper-thin flexible 'skin'. ScienceDaily. Retrieved December 11, 2018 from www.sciencedaily.com/releases/2013/05/130516105702.htm

Sungjun Park, Soo Won Heo, Wonryung Lee, Daishi Inoue, Zhi Jiang, Kilho Yu, Hiroaki Jinno, Daisuke Hashizume, Masaki Sekino, Tomoyuki Yokota, Kenjiro Fukuda, Keisuke Tajima, Takao Someya. (2018). Self-powered ultra-flexible electronics via nano-grating-patterned organic photovoltaics. Nature, 561 (7724): 516 DOI: 10.1038/s41586-018-0536-x

The Korea Advanced Institute of Science and Technology (KAIST). (2014, June 23). First demonstration of a selfpowered cardiac pacemaker. ScienceDaily. Retrieved December 11, 2018 from www.sciencedaily.com/releases/2014/06/140623120316.htm

University of California - San Diego. (2018, September 12). Wearable ultrasound patch monitors blood pressure deep inside body. ScienceDaily. Retrieved December 11, 2018 from www.sciencedaily.com/releases/2018/09/180912202421.htm

Wikipedia. (2018, November 25). Electrocardiography. In Wikipedia. Retrieved December 11, 2018, from https://en.wikipedia.org/wiki/Electrocardiography 